Can the Perceived Barriers to Psychological Treatment Scale be used to investigate treatment barriers among females with disordered and non-disordered eating behaviours? 


\begin{abstract}
There is a lack of psychometrically sound instruments to assess treatment barriers among individuals with disordered eating behaviours. This study examined the factor structure and psychometric properties of the Perceived Barriers to Psychological Treatment scale (PBPT; Mohr et al., 2010) among a sample of individuals with disordered eating behaviours.

Participants were 708 females aged 14 years and older who completed an online survey. The sample was randomly divided in two for the conduct of exploratory (EFA) and confirmatory (CFA) factor analyses. EFA suggested a seven-factor structure retaining 24 of the original 27 items (variance explained $=60 \%, \alpha=.91$ ). Factors were stigma, participation restrictions, negative evaluation of treatment, lack of motivation, emotional concerns, access restrictions, and time constraints. To assess clinical sensitivity, we conducted a secondary EFA utilising only clinical cases from this sample, which supported the solution but suggested retaining 25 of the original 27 items (variance explained $=58 \%, \alpha=0.89$ ). The 25 -item, seven-factor solution was further supported by CFA with an independent sample. Construct validity was also supported. The study suggests that the instrument will provide clinicians and researchers with a valid and reliable method of assessing treatment barriers in disordered eating samples.
\end{abstract}

Keywords: Barriers to care; barriers to treatment; eating disorder; help-seeking; treatment engagement 


\section{Can the Perceived Barriers to Psychological Treatment Scale be used to investigate treatment barriers among females with disordered and non-disordered eating behaviours?}

\section{Introduction}

Engagement with treatment services is problematic in disordered eating populations, in terms of help-seeking, dropout, and relapse prevention strategies (Guarda, 2008; Mahon, 2000). The proportion of individuals with a clinical eating disorder who access treatment in a single year is considerably less (19-36\%) than the proportion of individuals suffering other types of mental health problems (35-41\%; Cachelin and Striegel-Moore, 2006; Hart et al., 2011; Vanheusden et al., 2008). In addition, treatment for an eating disorder is sought an average of 10-15 years after the onset of their illness compared to an average 8.2 year delay in those with mood or anxiety disorders (Oakley et al., 2006; Thompson et al., 2004). There is a need to improve help-seeking in this population, and for that help-seeking to occur earlier in the disorder progression, including when symptoms are sub-clinical.

Failure to engage in treatment may be understood in terms of barriers to care (Hart et al., 2011; Innes et al., 2016). Instruments that systematically assess barriers to accessing specialised mental health services are important to understanding the specific factors that prevent individuals accessing and/or receiving treatment. A systematic review of the measurement of barriers to care among disordered eating populations identified shame/ stigma, poor mental health literacy, perceived need for treatment, unhelpful past treatment experiences, fear of change, low motivation, service restrictions, and cost as being key barriers inhibiting engagement with services (Innes et al., 2016). However, it was also revealed that there are very few psychometrically sound and appropriate scale-based instruments to adequately measure treatment barriers (Innes et al., 2016). Methods used to date have primarily used qualitative or checklist methods (e.g., Cachelin et al., 2001; Evans et 
al., 2011; Hepworth and Paxton, 2007; Meyer, 2001), which do not allow for standardised investigations or comparisons across individuals or populations. Furthermore, the only scalebased instrument available in the area of eating disorders (Cachelin et al., 2006) does not enable the relative influence of each barrier to be assessed and lacks any data on the psychometric properties of the instrument (Innes et al., 2016).

Within the broader treatment literature, the Perceived Barriers to Psychological Treatment (PBPT) scale (Mohr et al., 2010) has been widely used and has demonstrated sound psychometric properties for the accurate and comprehensive assessment of treatment barriers. It was designed to measure barriers toward attending weekly counselling or therapy for emotional or health (e.g. smoking cessation) problems. The PBPT has previously been used to assess treatment barriers (such as cost, stigma, availability of services, etc.) in areas such as the treatment of depression (e.g. Casey et al., 2014) and among a Veteran's sample with mixed mental health needs (Pfeiffer et al., 2016). However, to the authors' knowledge (and according to citing articles of the PBPT), the scale has yet to be used or validated for individuals with eating, weight, or body shape concerns. The purpose of the current study was to examine the psychometric properties of the PBPT among females with a range of eating behaviours and symptoms.

\subsection{The PBPT}

The PBPT (Mohr et al., 2010) has demonstrated excellent psychometric properties in assessing barriers specifically toward psychological treatment. This is particularly important given that rates of psychotherapy attendance for a range of mental health problems have declined over the past 40 years, despite a corresponding increase in receiving treatment for a mental health disorder (Mackenzie et al., 2014). This finding reflects a shift towards pharmacological interventions with general practitioners becoming an increasingly preferred professional source of help (Mackenzie et al., 2014; Olfson and Marcus, 2010; Reavley et al., 
2011). Such findings are of particular concern in the area of eating disorders given the demonstrated efficacy of cognitive behavioural treatment for Bulimia Nervosa $(\mathrm{BN})$ and Binge-Eating Disorder (BED) over and above pharmacological interventions (Fairburn and Harrison, 2003; Grilo et al., 2005). There is also a lack of evidence for the efficacy of pharmacological approaches in the treatment of Anorexia Nervosa (AN) (Zhu and Walsh, 2002).

Another important attribute of the PBPT is that it enables assessment of the degree of difficulty that each individual barrier may cause, allowing greater information to be gathered about barriers than by checklist approaches. Lastly, individuals can complete the PBPT at any stage of the help-seeking process and regardless of treatment history or type of mental health problem. For these reasons, the PBPT may prove useful in the assessment of treatment barriers in eating disorder populations.

\subsection{The current study}

The aim of this study was to test the utility of the PBPT for face-to-face psychological treatments for weight, shape, and eating behaviours in a mixed sample, inclusive of those meeting criteria for a clinical diagnosis of eating disorder, and those with concerns around their eating behaviours. The recruitment strategy employed was specifically designed so that women in the sample were similar on the presence of these concerns, regardless of whether or not they met clinical criteria. This approach is consistent with a continuum view of psychopathology and disordered eating (Ahmed et al., 2012; Garner et al., 1983; Gleaves et al., 2000) rather than categorical, and allowed for the scale to be tested in a sample representing a range of eating and weight concerns, not just among those with concerns of pathological level. This approach is also consistent with previous sampling approaches in the help-seeking and barriers literature (e.g., Fairburn, 2008; Gulliver et al., 2010; Mackenzie et al., 2007), and the original PBPT development study (Mohr et al., 2010). Furthermore, 
although the current study utilised a cross sectional design, longitudinally it is not uncommon for individuals with eating disorders to shift between clinical, subclinical, and non-clinical symptomology (Herzog et al., 1999). As such, although clinical status was used to describe the sample and allow judgment on representativeness, it was not intended for use in the analytical approach. The sample was however limited to females, due to the disproportionate representation of females across all categories of eating disorders and the resulting difficulty in recruiting an adequate sample size of males (Andersen and Holman, 1997; Wright et al., 2009). We examined the structural validity of the scale, as modified to focus on psychological barriers to treatment for concerns around eating behaviours, as well as explored construct validity through assessing convergent and divergent validity. In addition, the internal consistency of the PBPT was examined. Finally, as the PBPT allows for the examination of the relative influence of barriers to treatment, an explorative comparison of barriers (between identified subscales) was also planned.

Construct validity was tested by comparing the PBPT to items on the General Help Seeking Questionnaire (GHSQ) relating to intentions to seek help from a mental health professional, via online treatment, and a global "I would not seek help" item. Our expectation was that intentions to seek help and perceived barriers to accessing treatment are related but discrete constructs. For many, intentions to seek help are likely to be inversely related to the number of perceived barriers. However, some may identify few barriers to accessing treatment but have no intention to seek help, while some may have a strong intention to seek help but feel there are currently too many barriers to doing so. Therefore, we expect only a small to moderate correlation, suggesting discriminant validity of the PBPT and supporting the notion that PBPT responses reflect barriers to treatment as discrete from intentions to seek help. 
Assessment of depression is also important when examining possible treatment barriers, as depression is co-morbid in nearly $50 \%$ of individuals with eating disorders (Sullivan, 1995). Further, depression has also been identified as a barrier to treatment seeking (Mohr et al., 2006), which may influence ratings of other perceived barriers. To test whether ratings on the PBPT could be discriminated from severity of depressive symptoms, the relationship between treatment barriers (PBPT scores) and depressive symptoms were examined using scores from the Patient Health Questionnaire (PHQ-2).

\section{Method}

\subsection{Participants}

The study was conducted as an online survey and was advertised through information websites and forums regarding eating behaviours, among undergraduate university students, and at local exercise centres. The survey description was targeted at individuals who had shape, weight, and/or eating concerns, but was not restricted to individuals with an eating disorder, but was limited to females. Further, the age was restricted to females who were 14 years or older, which allowed for participation without parental consent.

The survey was accessed by 901 individuals, with 78.6\% $(n=708)$ of respondents completing the survey and 193 incomplete responses removed prior to analyses. The final sample comprised 708 females, ranging between the ages of 14 and 63 years $(M=21.79 ; S D$ =7.05). Participants were predominantly Caucasian $(n=576,81.36 \%)$, single $(n=563$, $79.52 \%)$, students $(n=530,74.86 \%)$ or working $(n=135,19.07 \%)$, and living in metropolitan areas $(n=564,79.66 \%)$. Of the retained responses, $288(40.7 \%)$ were sourced from the local community, $278(39.3 \%)$ were recruited through the host university, and 142 $(20.1 \%)$ were international respondents.

Based on the Questionnaire for Eating Disorder Diagnoses (Q-EDD) scores, most respondents in the analysed sample $(n=381,53.81 \%)$ were in the clinical range for an eating 
disorder, 95 (13.42\%) were in the subclinical range, and $232(32.77 \%)$ were in the nonclinical range. Of those in the clinical range, subcategories were: AN (20.11\%), BN (34.32\%), BED (3.22\%), and Other Specified Feeding or Eating Disorder (OSFED, 42.36\%). Although the clinical and sub-clinical groups were over-represented in the current sample (when compared to typical community sampling), it was deemed this was likely the result of the targeted recruitment strategy utilised. That is, the recruitment specifically called for females with shape, weight, or eating concerns, and as such, these individuals were more likely to self-select into the study than those without such concerns. As such, a distinction should also be made that the non-clinical group is not equivalent to a symptom or concern absent group. Rather, this group represents individuals who, by nature of the study focus and recruitment strategy, identified with some level of concern regarding eating, weight, or shape that did not reach clinical or sub-clinical thresholds. That is, although concerns may be present, they were not symptomatic at the time of assessment.

\subsection{Measures}

2.2.1. PBPT (Mohr et al., 2010). The PBPT is a self-report scale that addresses factors that may interfere with an individual receiving weekly psychological treatment. The PBPT consists of 25 items each representing a different barrier, for example "The cost of therapy would make it to attend weekly therapy". Each item is rated on a 5-point scale of perceived difficulty, ranging from 1 (Impossible to attend) to 5 (Not a problem).

The construct validity of the PBPT has been assessed using exploratory and confirmatory factor analysis, which yielded eight subscales: stigma (seven items), lack of motivation (two items), emotional concerns (three items), negative evaluation of therapy (four items), misfit of therapy to needs (four items), time constraints (two items), participation restrictions (four items), availability of services (two items), and cost (one item) with four items cross loading onto two scales (Mohr et al., 2010). Internal consistency for the 
subscales was found to be adequate to good $(\alpha=0.71-0.89)$, and excellent for the total scale $(\alpha=0.92)$. The scale also demonstrated strong predictive validity. The scoring approach outlined by Mohr et al. (2010) involves summing item to yield subscale scores and a total PBPT score which ranges between 0 and 125 (Mohr et al., 2010).

For the current study, the original 27 items factor analysed during development of the PBPT were incorporated. As we were exploring the utility of the instrument relating to treatment for a specific area of concern (shape, weight and eating behaviours) rather than general psychological treatment, the two items removed during the original measure development may have had increased validity in this context, and we were interested in exploring whether the factor structure from the original would hold across different treatment contexts.

To ensure that the PBPT would be relevant to specific barriers to receiving psychological treatment for problems with shape, weight and/or eating concerns, the PBPT instructions were adapted to ensure that the treatment in which barriers were assessed referred to receiving psychological treatment for problems with shape, weight, or eating concerns. Furthermore, the original PBPT instrument referred to either counsellor or therapist, thus to avoid confusion between these sources of professional help the term "counsellor" was eliminated from the original instructions.

2.2.2. The General Help Seeking Questionnaire (GHSQ; Wilson et al., 2005). The GHSQ measures individuals' future help-seeking behavioural intentions and can be adapted to any type of problem in addition to specific health sources. Participants rate the likelihood of seeking help from each source on 7-point Likert scales ranging from 1 (Extremely unlikely) to 7 (Extremely likely). Higher scores indicated higher intentions to seek treatment. The GHSQ has adequate internal consistency $(\alpha=0.70-0.83)$, test-retest reliability $(0.86-0.88$, 
over a three week period; Wilson et al., 2005), discriminant validity, and strong convergent and predictive validity. Internal consistency in the current study was acceptable $(\alpha=0.73)$.

Participants were asked about seeking help specifically for problems with weight, body shape, and/or eating behaviours. Of the ten items from the original questionnaire, three were of particular relevance to this study: standard treatment from a mental health professional, online treatment, and "I would not seek any help". The remaining items related to informal or medical (rather than psychological) forms of help-seeking and were not of interest for the current study.

2.2.3. Patient Health Questionnaire for Depression (PHQ-2; Kroenke et al., 2003) . The PHQ-2 is a brief screener for depression that uses the first two items from the PHQ-9 (Kroenke et al., 2001). Participants endorse the degree to which they were affected by the problem (e.g., 'little interest or pleasure in doing things') over the last two weeks, from 0 (Not at All) to 3 (Nearly Everyday), with total scores ranging from 0-6. The PHQ-2 has demonstrated sensitivity and specificity in detecting major depressive disorder, convergent validity with other measure of depression, and high internal consistency. For the present study, the PHQ-2 yielded a high internal consistency (Cronbach's $\alpha=0.90$ ).

2.2.4. Q-EDD (Mintz, O'Halloran, Mulholland, Schneider, 1997). The Q-EDD is a 50-item self-report questionnaire that was developed using the DSM-IV-TR criteria for eating disorders (Mintz et al., 1997). Items assess presence ('yes' or 'no') and frequency (scale ranging 1-6, example anchors 'daily' to 'once a month') of a range of eating and weight related behaviours (e.g. "Do you make yourself vomit to prevent weight gain?”). The Q-EDD uses branch tree logic to categorise individuals into two broad categories: eating disordered and non-eating disordered. The eating disorder category comprises: AN, BN, and four types of EDNOS: (a) Sub-threshold BN, (b) Menstruating AN, (c) Non-bingeing BN, and (d) BED. The non-eating disordered category is divided into asymptomatic (no disordered eating 
behaviours) or symptomatic (report eating disorder behaviours but do not meet diagnostic criteria). The Q-EDD has demonstrated adequate convergent and discriminant validity, interrater reliability, and specificity and sensitivity (Mintz et al., 1997; Thelen et al., 1991; Garner and Garfinkel, 1979). For the current study, minor modifications to the pre-existing categories on the Q-EDD were made in alignment with the development of the DSM-5. In addition, amendments to the coding were made to further strengthen the accuracy of specific diagnoses. For example, the initial Q-EDD coding was missing some key features of AN. AN was missing reference to restriction of caloric intake and instead relied on BMI, so this was adjusted to also include dieting and/or fasting behaviours for at least three months.

\subsection{Procedure}

Ethical approval was obtained from the institution's ethics review committee. The measures for the current study formed part of a larger online survey addressing similar themes, and was administered online, taking approximately 30-45 minutes to complete. At the end of the survey participants were provided with a list of possible referrals to eating disorder organisations/services within various countries. For the non-student sample, participants had the option to enter into a prize draw to win 1 of 5 gift vouchers $(1 \mathrm{x} \$ 100$ and 4 x \$50). Undergraduate student participants received partial course credit for participation. Participants' identifying details were not linked to responses for either sample. The survey was set to prevent repeat participation by the same person using cookies (Eysenbach, 2004).

\subsection{Statistical approach}

As this was not a replication study, it was considered appropriate to first conduct an EFA to examine the number of factors emerging in our slightly modified PBPT. To crossvalidate the factor structure, a confirmatory factor analysis (CFA) was subsequently conducted. The full dataset was split into half, with 354 assigned at random to the EFA and the rest $(n=354)$ used for the CFA. 
All descriptive and preliminary analyses were conducted using $R$ (R Core Team, 2016). Internal consistency was assessed using Cronbach's alpha and corrected item-total correlations. Pearson correlation coefficients were computed to examine construct validity. Exploratory factor analysis was conducted using the psych package in $R$ (Revelle, 2016), while Mplus v7.4 (Muthén and Muthén, 2012) was used for confirmatory factor analysis. To assess model fit in EFA, interpretability of the factor structure was a key consideration (Schmitt, 2011) along with TLI and RMSEA as secondary indicators. Items with factor loadings and/or communalities $<0.30$ were deemed to perform poorly and considered as candidates for potential removal. For CFA, chi-square goodness-of-fit tests were conducted, however due to its sensitivity to sample size we also examined alternative fit indices including CFI, RMSEA and SRMR (Hu and Bentler, 1999).

\section{Results}

\subsection{Preliminary analyses}

Normality was investigated for both the EFA and CFA datasets through inspection of univariate distributions for each variable (skewness, kurtosis, and normality plots), Mardia's multivariate tests of skewness and kurtosis, and the Henze-Zirkler (1990) multivariate normality test, which compares the observed distribution to a hypothetical multivariate normal distribution. Both datasets demonstrated significant departure from multivariate normality $(\mathrm{HZ}=1.09, p=<.001$ for the EFA data, and $\mathrm{HZ}=1.18, p<.001$ for the CFA data), which we address below.

\subsection{Exploratory Factor Analysis}

Visual inspection of multivariate outliers was first conducted by plotting robust Mahalanobis distances against quantiles of the Chi-square distribution (Yuan and Zhong, 2008). Removal of the six cases with the highest Mahalanobis distance scores improved multivariate normality based on visual inspection of the plot. Although outlier removal did 
not significantly improve the overall multivariate normality of the distribution statistically, the interpretability of the factor structure in the subsequent EFA improved; thus they were excluded leaving a total of 342 cases. Sample sizes above 300 are generally recommended for EFA, or a minimum of five cases per variable (Stevens, 2002). Therefore, the remaining sample was considered sufficiently large for EFA.

To assess suitability for factor analysis, the Kaiser-Meyer-Olkin measure of sampling adequacy (MSA) was computed. An overall MSA of 0.89 (0.68 to 0.95 for individual items) suggested the data was suitable for EFA (Hutcheson and Sofroniou, 1999). This assumption was supported by a significant Barlett's Test of Sphericity $(p<.001)$.

To determine the number of factors to retain in EFA we used parallel analysis (PA), typically recommended as a superior approach relative to traditional methods such as visual examination of the scree plot or eigenvalues $>1$ (Hayton et al., 2004). PA suggested retaining eight factors, however as interpretability of the factor structure is also an important consideration (Ford et al., 1986) we examined six, seven and eight-factor solutions, with the seven-factor solution emerging as the cleanest and most interpretable.

Factor extraction was conducted using minimum residual estimation (MINRES), an unweighted least squares approach comparable to Principal Axis factoring and suitable for data that is multivariate non-normal (Zygmont and Smith, 2014). An oblique Geomin factor rotation was used to improve interpretability of the pattern matrix and allow for correlated factors.

The initial seven-factor solution explained $56 \%$ of the item variance, and had reasonable model fit, TLI $=0.905, \mathrm{RMSEA}=0.061$ [95\% CI: $0.051,0.066]$. Following review of the strongest loading items, factors were labelled as follows: (1) Stigma (concerns of judgment from self or others), (2) Participation restrictions (for example, physical conditions that would make it difficult to attend therapy), (3) Negative evaluation (for 
example, that therapy would not be helpful), (4) Lack of motivation (for example, motivation to make an appointment), (5) Emotional concerns (that attending therapy may result in difficult emotions), (6) Access to services (for example concerns of cost or availability), and (7) Time constraints (for example, getting time off work to attend therapy). Labels largely reflected the eight-factor structure identified in the original PBPT validation study (Mohr et al, 2010), with the exception of the factor Misfit of therapy to needs.

Follow-up of the initial rotated factor solution was conducted for three items $(2,14$, and 21) with both poor communality estimates $(<0.40)$ and no clear loading on a single factor. These were explored for substantive fit and interpretability of the factor structure if removed. Item 14 (Attending therapy is too self-indulgent) did not load strongly onto any of the factors and review of item content revealed a lack of clear substantive fit, and so it was removed. Item 2 (The responsibility of caring for loved ones) cross-loaded weakly onto Factors $2(0.41)$ and 5 (0.36). Although this item had some conceptual fit with both factors, its low communality $(0.32)$ and lack of a clear, unambiguous contribution to either one made it a suitable candidate for removal. This item was also identified as problematic by the scale developers and removed in the original PBPT validation study (Mohr et al., 2010). Finally, item 21 (My problems are not severe enough for therapy) had only a weak loading on Factor 1: Stigma (0.36), and had poor communality (0.26) and item-total correlation $(r=0.48)$. This item loaded onto an eighth factor in the original validation study (Misfit of therapy to needs) which did not emerge in our data, and as it appeared to reflect a somewhat different concept to stigma, was removed.

Model fit indices improved for the final seven-factor model with items 2, 14 and 21 removed $(\mathrm{TLI}=0.936, \mathrm{RMSEA}=0.054[95 \% \mathrm{CI}: 0.042,0.061])$, and a clear factor structure was retained with no cross-loading items. The final model accounted for $60 \%$ of the item variance. Table 1 shows factor loadings based on the rotated pattern matrix, along with 
corrected item-total correlations and internal consistency (Cronbach's alpha) estimates for subscales based on factor sum scores. Internal consistency was good for most subscales ( $\alpha$ range $=0.67-0.89)$, with Factors 6 and 7 in the borderline range $(0.69$ and 0.67 respectively). Overall internal consistency was high $(\alpha=0.91)$.

\section{[Approximate location of Table 1]}

\subsection{Sensitivity to clinical status}

Although all participants identified with some level of eating, weight, or shape concerns, we were cognisant of whether the factor structure was sensitive to clinical status, and thus influenced by the mix of clinical and community participants in our sample. To investigate, we conducted a secondary exploratory analysis of the factor structure using a clinical subset of our EFA sample, restricted to only those scoring in the clinical or subclinical range on the Q-EDD $(n=250)$. Using a similar process as outlined above, we removed nine cases identified as multivariate outliers or with no score variance $(n=241)$, followed by parallel analysis and EFA. Results of this second factor analysis are shown in Table 2.

Overall, we found a similar structure as before, with seven factors retained and identical factor labels. Items 2 and 21 were again removed for the reasons described earlier. However, we found two differences at the item level. First, item 27 (Concerns about medical or insurance records of therapy) was retained in the original EFA on the Stigma factor with a loading of 0.43 , however this loading dropped to 0.34 in the clinical EFA, indicating poorer fit overall across all factors. This represents a minor change in the overall factor loading, and may be attributable to random error arising from the use of clinical subset of our broader sample. Nonetheless, we acknowledge this discrepancy may also reflect underlying differences between clinical and community individuals regarding their views around medical and insurance records. We theorise two possible causes here: first, individuals with more 
severe problems may have greater prior experience with clinical services and thus more exposure to record-keeping processes, which may have a normalising effect that reduces its impact on feelings of stigma. Alternatively, clinical individuals may have more concerns around record keeping in general, resulting in a more restricted range of response variance that lowers its factor weighting. We were not able to test these theories in the current research, but highlight these as areas of interest for possible future work.

The second item-level difference was item 14 (Attending therapy feels too selfindulgent). This item was removed from the original EFA due to poor loading across all factors. In our clinical re-analysis, it was found to have a significant loading of 0.53 onto the Negative evaluation factor, with no strong cross-loadings. This suggests this item may be more relevant to how people with clinical levels of concerns negatively evaluate therapy; for example, these individuals may experience more shame, guilt, or negative self-talk than nonclinical individuals, leading to stronger endorsement of this cognitive perception.

For the revised clinical EFA model, model fit was adequate $(\mathrm{TLI}=0.906, \mathrm{RMSEA}=$ 0.063 [95\% CI: $0.047,0.091]$ ), accounting for $58 \%$ of the item variance with adequate internal consistency across factors (see Table 2).

\section{[Approximate location of Table 2]}

\subsection{Confirmatory factor analysis.}

Confirmatory factor analysis was conducted to cross-validate the factor structure identified through EFA using the remaining sample of 354 cases. Non-normality of the dataset was addressed in Mplus by using the MLR estimator, which computes robust standard errors (Muthén and Muthén, 2012).

We first tested the factor structure identified from the initial EFA, with items 2, 14, and 21 removed. The chi-square test of model fit for this 24 -item, seven-factor solution was significant, $\chi^{2}(231)=499.53, p<.001$, although alternative fit indices indicated the model had 
adequate fit, CFI $=0.924$, RMSEA $=0.057$ [95\% CI: 0.050, 0.064], SRMR $=0.058$. We then conducted a follow-up CFA with item 14 retained in the scale, based on our second EFA with the clinical subset of participants which suggested this item may have utility for these individuals. Although there was a slight decline in fit, overall fit indices remained adequate, $\chi^{2}(254)=587.65, p<.001, \mathrm{CFI}=0.912, \mathrm{RMSEA}=0.061[95 \% \mathrm{CI}: 0.054,0.067], \mathrm{SRMR}=$ 0.058. Given this, we prefer this second model as it retains more of the original PBPT items, and may have greater utility for individuals with clinical levels of difficulty around eating behaviours.

Further examination of modification indices revealed incremental improvements could be achieved through correlating residual errors or cross-loading items, but as these steps would have required substantial modifications to the measurement model in the absence of a strong theoretical rationale, we opted for the more parsimonious solution and retained the seven-factor model without further changes. Figure 1 shows standardized loadings and residual estimates for the final CFA measurement model with 25 items.

\section{[Approximate location of Figure 1]}

\subsection{Construct validity of the PBPT}

The GHSQ item 'I would not seek help from anyone' had a significant moderate positive correlation with the PBPT Total score as hypothesised $(r=0.41, p<.01)$. At the subscale level, this GHSQ item had the strongest relationship with the Stigma subscale of the PBPT $(r=0.45, p<.01)$. The total PBPT score also showed a weak but significant negative correlation with scores on the GHSQ item referring to intentions to seek help from a mental health professional $(r=-0.19, p<.05)$, as well as the overall GHSQ Total score computed from the three individual items $(r=-0.28, p<.05)$. There was however no relationship between the PBPT Total score and reported intentions to seek online treatment $(r=0.00, p=$ $1.00)$. 
A moderate correlation was found between the PBPT Total scores and depressive symptoms as measured by the PHQ-2 $(r=0.39, p<.001)$, suggesting a moderate relation between depression and perceived barriers to accessing psychological treatment. The strength of the correlation suggests that despite this relationship, perceived barriers to accessing psychological treatment for shape, weight or eating concerns are likely influenced by other discrete psychological mechanisms. Correlations between the PBPT and measures of validity are displayed in Table 3.

\section{[Approximate location of Table 3]}

\subsection{Comparison of subscale scores}

To explore the potential clinical utility of the PBPT we performed a comparison of subscale scores to determine whether particular barriers emerged as more critical than others in the current sample. As there were different numbers of items per subscale we modified the sum score technique outlined by Mohr et al. (2010) to utilize a mean subscale score (i.e. sum score divided by number of items), thus allowing for comparison between subscales. Table 4 shows mean scores and standard deviations for each subscale, with Access to services emerging as the highest-rated perceived barrier, followed by Time constraints. Participation restrictions had the lowest mean rating. As subscale scores were not from independent samples, a univariate within-subjects ANOVA was computed with subscale as the withinsubjects predictor and mean score as the dependent variable. Results showed a significant overall difference between subscales in terms of mean scores, $F(4.69,1656.40)=108.29, p<$ .001 (Greenhousse-Geisser sphericity correction applied). Individual follow-up $t$-tests using Dunnett adjustment for multiple correlated comparisons were conducted to compare each subscale with the subsequent, next highest-scoring subscale. Post hoc tests showed no significant difference in mean scores between the two highest-rated subscales (Access to services, Time constraints), while these were both rated significantly higher than all other 
subscales. There were no significant differences between Stigma, Lack of motivation, and Negative evaluation, while Emotional concerns and Participation restrictions were given significantly lower ratings than all other subscales.

\section{[Approximate location of Table 4]}

\section{Discussion}

The aim of this study was to determine if the PBPT can be used to assess treatment barriers in individuals with a range of eating behaviours (non-disordered and disordered). The psychometric properties of the PBPT were examined, focusing on the construct validity of the scale and internal consistency. Evidence for the validity and reliability of this instrument supports the use of the PBPT to investigate and accurately capture barriers in a mixed sample of individuals with a range of eating behaviours. The response format of this scale-based instrument allows the relative influence of each barrier to be determined thereby permitting the greatest understanding of barriers. The only other scale-based instrument available in the area of eating disorders (Cachelin et al., 2006) does not enable the relative influence of each barrier to be assessed and lacks data on the psychometric properties of the instrument (Innes et al., 2016), making the PBPT a particularly useful tool. Furthermore, the PBPT captures barriers specific to psychological treatment, which is important given the low rates of helpseeking from this professional source by individuals with eating disorders (Mond et al., 2009; Mond et al., 2008).

The PBPT was originally developed using a sample of primary care patients (Mohr et al., 2010), with the measure yet to be validated on a sample with disordered and nondisordered eating behaviours. The results of this study supported many of the original findings by Mohr et al. (2010) on the internal reliability and construct validity of the PBPT. However, an important difference in our study was that a clear seven-factor solution was found, initially retaining 24 of the original 27 items, and revised to 25 items after further 
examination within a subset of individuals falling in the clinical or subclinical range. No items loaded substantively onto more than one subscale, and the 25 -item factor structure was supported through confirmatory factor analysis with an independent sample, showing adequate fit and no correlated residual error terms or cross-loading items.

There were some discrepancies between our findings and the original analyses reported by Mohr et al. (2010), in which an eight-factor solution was retained with multiple items allowed to cross load onto different subscales. It is unclear whether these differences result from response characteristics related to eating behaviours versus general primary care, or alternatively whether a modified seven-factor solution may have produced a cleaner factor structure in the original PBPT study if explored further. Notably, our reduced seven-factor solution led to the discarding of two of the original PBPT items due to poor loadings, item 2 (The responsibility of caring for loved ones) and item 21 (My problems are not severe enough for therapy). Our decision to remove item 2 was supported by its removal in the original validation study, however item 21 loaded onto an eighth factor in the original study (Misfit of therapy to needs) that was not retained here. Perhaps an evaluation of this item within a strictly non-clinical sample (rather than a combined community and clinical sample), where lack of problem severity may be a greater barrier to help-seeking, would lead to stronger loading on one of the existing factors (e.g. Stigma). We were unable to perform a subgroup analysis containing only non-clinical individuals due to sample size limitations. However, we feel it is more likely that this item taps into a different construct regarding the perceived 'fit' of therapy, as identified within the original study. It is important to note that our decision to remove this item and the eighth factor in the present analyses are no reflection on the practical value of ascertaining whether therapy fits an individual's needs. Rather, their removal here suggests that the PBPT in its present form may not contain enough items with 
sufficient content validity to tap into this area, at least in the context of help-seeking for eating, shape, and weight concerns.

Further evidence of construct validity of the PBPT was found in our study. The scale showed a small but significant negative correlation $(r=-0.28)$ with overall help-seeking intentions, and intentions to seek help from a mental health professional $(r=-0.19)$.

Likewise, a significant positive correlation was found with the item "I would not seek help" $(r=0.40)$. These correlations are small to moderate in strength, suggesting the PBPT is able to discriminate between the related constructs of barriers to treatment and intentions to access treatment.

Surprisingly, the PBPT failed to produce any correlation with intentions to seek online treatment. We had expected that more barriers to accessing face-to-face treatment may have been related to lower intentions to access any form of treatment, online supports included. This was not the case in the current study, suggesting that identified barriers and intentions to access treatment via different treatment modalities may need to be considered on their own merits. This is further supported by a non-significant correlation between the GHSQ items assessing intentions to access online treatment versus a mental health professional. Many of the common barriers to accessing in-person therapy are less relevant to accessing online supports, reflected in our sample through these uncorrelated scores. These findings suggest further exploration around the measurement of barriers to accessing online supports in the current context is warranted.

As expected, the PBPT showed a positive moderate relationship with PHQ-2 scores. Mohr et al. (2006) also found depressive symptoms were associated with increased perceived barriers (total PBPT scores). These findings suggest that perceived barriers to treatment are related to, but not solely influenced by depressive symptoms, and that the PBPT is therefore suitable for assessing barriers in individuals with co-morbid mental health problems. In 
summary, these findings extend upon the original work by Mohr et al (2010), providing evidence of construct validity of the PBPT in the current sample.

An examination of subscale scores revealed the subscales of Access to Services (cost and availability of services) and Time Constraints (therapy interfering with daily tasks or getting time off work to attend therapy) as being the most highly endorsed barriers to treatment for shape/ weight/ eating concerns among the sample. This finding is consistent with the previous body of research, which has found these concerns to be prevalent among disordered eating populations (Innes et al., 2017). However, that these concerns were rated more highly than concerns such as Stigma and Negative Evaluation is surprising, given the importance often placed on these barriers in the literature (e.g. Becker et al., 2010; Cachelin and Striegel-Moore, 2006; Evans et al., 2011; Hepworth and Paxton, 2007). As this is the first study to utilise a scale that enables assessment of relative influence of barriers in this population, further research is required to support this finding. However, based on these results, future research may benefit from utilising innovative approaches to target the identified barriers, such as through education and information programs to disseminate flexible (and cost-effective) online intervention programs.

\subsection{Limitations and directions for future research}

Results of the current study should be considered within the context of a number of limitations. Firstly, a female only sample was utilised for practical and theoretical reasons. As such, results of the current study may not be able to generalise to males with eating, weight, or shape concerns. Furthermore, consistent with the original measure, items in the PBPT focus on barriers to attending weekly, face-to-face psychological treatment. The measure may not therefore capture barriers to alternate treatment styles (such as online therapies) or treatment delivered at different intervals (e.g. monthly). Future research is required to determine the validity and utility of the scale in these different contexts and samples. 
Finally, it is conceivable that perceived barriers may vary depending on the age of the respondent, an issue which is not explored in our study. Younger girls (e.g. ages $14-16$ ) may experience unique barriers that prevent help-seeking, such as access to transportation, cost of therapy, or a reluctance to disclose issues to parents, which are less relevant for older women. While the PBPT may provide clinical utility for exploring such age-based differences, an important prior step (beyond the scope of this study) would be ensuring measurement properties are retained across ages through multigroup tests of measurement invariance (Milfont \& Fischer, 2015).

\subsection{Conclusions}

Psychometric analyses reported in this study suggest that the modified version of the PBPT is suitable for use in populations with difficult eating behaviours, and performs well across a mixed sample of clinical and non-clinical (but concerned) individuals. The PBPT may provide a useful method of assessing treatment barriers in these samples, although follow-up evaluation assessing item functioning within independent clinical and community samples may be beneficial given some marginal differences in item responding in the present study. Nonetheless, the adequate assessment of barriers to help-seeking is of primary importance in both clinical and non-clinical populations, given the low and delayed rates of help-seeking typically observed. Adequate assessment will then enable clinicians and researchers to design and assess interventions that may help individuals overcome these barriers. 


\section{References}

Ahmed, A.O., Green, B.A., Buckley, P.F., McFarland, M.E., 2012. Taxometric analyses of paranoid and schizoid personality disorders. Psychiatry Research 196 (1), 123-132.

Andersen, A. E., Holman, J. E., 1997. Males with eating disorders: Challenges for treatment and research. Psychopharmacology bulletin, 33(3), 391-397.

Becker, A. E., Arrindell, A. H., Perloe, A., Fay, K., Striegel-Moore, R. H., 2010. A qualitative study of perceived social barriers to care for eating disorders: Perspectives from ethnically diverse health care consumers. International Journal of Eating Disorders, 43(7), 633-647.

Cachelin, F. M., Rebeck, R., Veisel, C., Striegel-Moore, R. H., 2001. Barriers to treatment for eating disorders among ethnically diverse women. International Journal of Eating Disorders, 30(3), 269-278.

Cachelin, F. M., Striegel-Moore, R. H., 2006. Help seeking and barriers to treatment in a community sample of Mexican American and European American women with eating disorders. International Journal of Eating Disorders, 39(2), 154-161.

Casey, L. M., Wright, M. A., Clough, B. A., 2014. Comparison of perceived barriers and treatment preferences associated with internet-based and face-to-face psychological treatment of depression. International Journal of Cyber Behavior, Psychology and Learning, 4, 16-22.

Evans, E. J., Hay, P. J., Mond, J., Paxton, S. J., Quirk, F., Rodgers, B., . . Sawoniewska, M. A., 2011. Barriers to help-seeking in young women with eating disorders: A qualitative exploration in a longitudinal community survey. Eating Disorders: The Journal of Treatment \& Prevention, 19(3), 270-285. 
Eysenbach, G., 2004. Improving the quality of Web surveys: the Checklist for Reporting Results of Internet E-Surveys (CHERRIES). Journal of Medical Internet Research, $6(3), \mathrm{e} 34$.

Fairburn, C. G., 2008. Cognitive behavior therapy and eating disorders. Guilford Press: New York, NY.

Fairburn, C. G., Harrison, P. J., 2003. Eating disorders. The Lancet, 361(9355), 407-416.

Ford, J. K., MacCallum, R. C., \& Tait, M. (1986). The application of exploratory factor analysis in applied psychology: A critical review and analysis. Personnel Psychology, 39(2), 291-314. http://doi.org/10.1111/j.1744-6570.1986.tb00583.x

Garner, D. M., Garfinkel, P. E., 1979. The Eating Attitudes Test: An index of the symptoms of anorexia nervosa. Psychological medicine, 9(2), 273-279.

Garner, D.M., Olmsted, M.P., Garfinkel, P.E., 1983. Does anorexia nervosa occur on a continuum? Subgroups of weight-preoccupied women and their relationship to anorexia nervosa. International Journal of Eating Disorders.

Gleaves, D.H., Lowe, M.R., Green, B.A., Cororve, M.B., Williams, T.L., 2000. Do anorexia and bulimia nervosa occur on a continuum? A taxometric analysis. Behavior Therapy $31(2), 195-219$.

Grilo, C. M., Masheb, R. M., Wilson, G. T., 2005. Efficacy of cognitive behavioral therapy and fluoxetine for the treatment of binge eating disorder: a randomized double-blind placebo-controlled comparison. Biological Psychiatry, 57(3), 301-309.

Guarda, A. S., 2008. Treatment of anorexia nervosa: Insights and obstacles. Physiology \& Behavior, 94(1), 113-120. doi:http://dx.doi.org/10.1016/j.physbeh.2007.11.020

Gulliver, A., Griffiths, K. M., Christensen, H., (2010). Perceived barriers and facilitators to mental health help-seeking in young people: A systematic review. BMC Psychiatry, 10, 113-121. 
Hart, L. M., Granillo, M. T., Jorm, A. F., Paxton, S. J., 2011. Unmet need for treatment in the eating disorders: A systematic review of eating disorder specific treatment seeking among community cases. Clinical Psychology Review, 31, 727 - 735.

Hayton, J. C., Allen, D. G., \& Scarpello, V. (2004). Factor Retention Decisions in Exploratory Factor Analysis: A Tutorial on Parallel Analysis. Organizational Research Methods, 7(2), 191-205. http://doi.org/10.1177/1094428104263675

Henze, N., \& Zirkler, B. (1990). A class of invariant consistent tests for multivariate normality. Communications in Statistics - Theory and Methods, 19(10), 3595-3617. http://doi.org/10.1080/03610929008830400

Hepworth, N., Paxton, S. J., 2007. Pathways to help-seeking in bulimia nervosa and binge eating problems: A concept mapping approach. International Journal of Eating Disorders, 40(6), 493-504.

Herzog, D.B., Dorer, D. J., Keel, P. K. Selwyn, S. E., Ekeblad, E. R., Flores, A. T., Greenworrd, D. N., Burweel, R. A., Keller, M. B., 1999. Recovery and relapse in anorexia and bulimia nervosa: A 7.5-year follow-up study. Journal of the American Academy of child \& Adolescent Psychiatry, 38, 829-837.

Holm, S. (1979). A simple sequentially rejective multiple test procedure. Scandinavian Journal of Statistics, 6(2), 65-70. Retrieved from http://www.jstor.org/stable/4615733

Hu, L., \& Bentler, P. M. (1999). Cutoff criteria for fit indexes in covariance structure analysis: Conventional criteria versus new alternatives. Structural Equation Modeling: A Multidisciplinary Journal, 6(1), 1-55. http://doi.org/10.1080/10705519909540118

Hutcheson, G. D., Sofroniou, N., 1999. The multivariate social scientist: Introductory statistics using generalised linear models. London, UK: Sage. 
Innes, T., Casey, L. M., Clough, B. A., 2017. Assessing Treatment Barriers in Eating Disorders: A Systematic Review. Eating Disorders: Journal of Treatment and Prevention, 25, 1-21.

Kroenke, K., Spitzer, R. L., Williams, J. B., 2001. The Phq-9. Journal of General Internal Medicine, 16(9), 606-613.

Kroenke, K., Spitzer, R. L., Williams, J. B., 2003. The Patient Health Questionnaire-2: validity of a two-item depression screener. Medical care, 41(11), 1284-1292.

Mackenzie, C. S., Erickson, J., Deane, F. P., Wright, M., 2014. Changes in attitudes toward seeking mental health services: A 40-year cross-temporal meta-analysis. Clinical Psychology Review, 34(2), 99-106.

Mackenzie, C.S., Geokoski, W. L., Knox, V. J., 2007. Age, gender and the underutilization of mental health services: The influence of help-seeking attitudes. Aging \& Mental Health, 10, 574-582.

Mahon, J., 2000. Dropping Out from Psychological Treatment for Eating Disorders: What are the Issues? European Eating Disorders Review, 8(3), 198-216.

Meyer, D. F., 2001. Help-seeking for eating disorders in female adolescents. Journal of College Student Psychotherapy, 15(4), 23-36.

Milfont, T. L., \& Fischer, R. (2015). Testing measurement invariance across groups: applications in cross-cultural research. International Journal of Psychological Research, 3(1), 111-130. doi:10.21500/20112084.857

Mohr, D. C., Hart, S. L., Howard, I., Julian, L., Vella, L., Catledge, C., Feldman, M. D., 2006. Barriers to psychotherapy among depressed and nondepressed primary care patients. Annals of Behavioral Medicine, 32(3), 254-258. 
Mohr, D. C., Ho, J., Duffecy, J., Baron, K. G., Lehman, K. A., Jin, L., Reifler, D., 2010. Perceived barriers to psychological treatments and their relationship to depression. Journal of Clinical Psychology, 66(4), 394-409.

Mond, J. M., Hay, P., Darby, A., Paxton, S. J., Quirk, F., Buttner, P., . . Rodgers, B., 2009. Women with bulimic eating disorders: When do they receive treatment for an eating problem? Journal of Consulting and Clinical Psychology, 77(5), 835-844.

Mond, J. M., Hay, P., Rodgers, B., Owen, C., 2008. Mental health literacy and eating disorders: What do women with bulimic eating disorders think and know about bulimia nervosa and its treatment? Journal of Mental Health, 17(6), 565-575.

Muthén, L. K., \& Muthén, B. O. (2012). Mplus User's Guide. Seventh Edition. Los Angeles, CA: Muthén \& Muthén.

Oakley Browne, M. A., Elisabeth Wells, J., Mcgee, M. A., 2006. Twelve-month and lifetime health service use in Te Rau Hinengaro: the New Zealand mental health survey. Australian and New Zealand Journal of Psychiatry, 40(10), 855-864.

Olfson, M., Marcus, S. C., 2010. National trends in outpatient psychotherapy. American Journal of Psychiatry, 167, 1456 - 1463.

Pfeiffer, P. N., Bowersox, N., Birgenheir, D., Burgess, J. Forman, J. Valenstein, M., 2016. Preferences and barriers to care following psychiatric hospitalization at two Veterans Affairs medical centers: A mixed methods study. The Journal of Behavioural Health Services \& Research, 43, 88-103.

R Core Team. (2016). R: A Language and Environment for Statistical Computing. Vienna, Austria. Retrieved from https://www.r-project.org/

Reavley, N. J., Yap, M. B., Wright, A., Jorm, A. F., 2011. Actions taken by young people to deal with mental disorders: findings from an Australian national survey of youth. Early Intervention in Psychiatry, 5(4), 335-342. 
Revelle, W. (2016). psych: Procedures for Psychological, Psychometric, and Personality Research. Evanston, Illinois. Retrieved from http://cran.r-project.org/package=psych Schmitt, T. A. (2011). Current Methodological Considerations in Exploratory and Confirmatory Factor Analysis. Journal of Psychoeducational Assessment, 29(4), 304321. http://doi.org/10.1177/0734282911406653

Stevens, J., 2002. Applied Multivariate Statistics for the Social Sciences, Fourth Ed. Mahwah, NJ: Lawrence Erlbaum Associates.

Sullivan, P. F., 1995. Mortality in anorexia nervosa. American Journal of Psychiatry, 152(7), 1073-1074.

Thelen, M. H., Farmer, J., Wonderlich, S., Smith, M., 1991. A revision of the Bulimia Test: The BULIT—R. Psychological Assessment: A Journal of Consulting and Clinical Psychology, 3(1), 119. doi: 10.1037/1040-3590.3.1.119

Thompson, A., Hunt, C., Issakidis, C., 2004. Why wait? Reasons for delay and prompts to seek help for mental health problems in an Australian clinical sample. Social psychiatry and psychiatric epidemiology, 39(10), 810-817.

Vanheusden, K., Mulder, C. L., van der Ende, J., van Lenthe, F. J., Mackenbach, J. P., Verhulst, F. C., 2008. Young adults face major barriers to seeking help from mental health services. Patient Education and Counseling, 73(1), 97-104. doi: 10.1016/j.pec.2008.05.006

Wilson, C. J., Deane, F. P., Ciarrochi, J., Rickwood, D., 2005. Measuring help-seeking intentions: Properties of the general help-seeking questionnaire. Canadian Journal of Counselling, 39(1), $15-28$.

Wright, F., Bewick, B. M., Barkham, M., House, A. O., Hill, A. J., 2009. Co-occurrence of self-reported disordered eating and self-harm in UK university students. British Journal of Clinical Psychology, 48(4), 397-410. 
Yuan, K.-H., \& Zhong, X. (2008). Outliers, Leverage Observations, and Influential Cases in Factor Analysis: Using Robust Procedures to Minimize Their Effect. Sociological Methodology, 38(1), 329-368. http://doi.org/10.1111/j.1467-9531.2008.00198.x

Zhu, A. J., Walsh, B. T., 2002. Pharmacologic treatment of eating disorders. Canadian Journal of Psychiatry, 47(3), 227-234.

Zygmont, C., Smith, M. R., 2014. Robust factor analysis in the presence of normality violations, missing data, and outliers: Empirical questions and possible solutions. Tutorials in Quantitative Methods for Psychology, 10(1), 40-55. Retrieved from http://www.tqmp.org/RegularArticles/vol10-1/p040/index.html 
Table 1. Summary of results from Exploratory Factor Analysis of the PBPT scale $(n=342)$

\begin{tabular}{|c|c|c|c|c|c|c|c|c|}
\hline & \multicolumn{7}{|c|}{ Factors } & \multirow{2}{*}{$\begin{array}{c}\text { Corrected } \\
\text { item-total } \\
\text { correlation }\end{array}$} \\
\hline & 1 & 2 & 3 & 4 & 5 & 6 & 7 & \\
\hline \multicolumn{9}{|l|}{ 1. Stigma } \\
\hline 23. Talking to someone about personal issues & 0.86 & 0.02 & 0.00 & -0.01 & 0.00 & -0.09 & 0.01 & 0.76 \\
\hline 24. Concern about being judged by a therapist & 0.79 & -0.05 & 0.11 & 0.01 & 0.03 & 0.05 & -0.04 & 0.81 \\
\hline 26. Accessing therapy means not able to solve own problems & 0.66 & 0.02 & 0.01 & -0.05 & 0.05 & -0.01 & 0.15 & 0.67 \\
\hline 22. Family and/or friends knowing about therapy & 0.61 & -0.01 & -0.11 & -0.02 & 0.00 & 0.10 & 0.07 & 0.56 \\
\hline 25. Do not think a therapist would truly care about me & 0.60 & -0.03 & 0.25 & 0.06 & 0.00 & 0.02 & -0.04 & 0.70 \\
\hline 20. Discomfort around someone seeing me emotional & 0.51 & 0.01 & -0.01 & 0.15 & 0.29 & 0.02 & -0.02 & 0.72 \\
\hline 27. Concerns about medical or insurance records of therapy & $\mathbf{0 . 4 3}$ & 0.06 & 0.02 & 0.02 & 0.03 & 0.16 & 0.06 & 0.54 \\
\hline \multicolumn{9}{|l|}{ 2. Participation restrictions } \\
\hline 8. Difficulties walking or getting around & 0.03 & 0.91 & -0.04 & -0.02 & 0.01 & -0.06 & 0.03 & 0.77 \\
\hline 9. Physical symptoms (fatigue, pain, breathing difficulties etc.) & -0.02 & 0.73 & 0.03 & 0.15 & 0.00 & 0.04 & 0.02 & 0.70 \\
\hline 10. Serious illness makes it hard to leave home & -0.01 & 0.72 & 0.20 & -0.04 & 0.01 & 0.02 & -0.04 & 0.69 \\
\hline \multicolumn{9}{|l|}{ 3. Negative evaluation } \\
\hline 12. Distrust of therapists & 0.16 & 0.02 & 0.76 & -0.04 & 0.06 & -0.01 & 0.03 & 0.75 \\
\hline 11. Bad past experiences & -0.04 & 0.04 & 0.71 & 0.12 & -0.04 & 0.07 & -0.03 & 0.65 \\
\hline 13. Would not expect therapy to be helpful & 0.22 & -0.02 & 0.57 & 0.02 & 0.01 & 0.00 & 0.07 & 0.65 \\
\hline \multicolumn{9}{|l|}{ 4. Lack of motivation } \\
\hline 19. Difficulty getting motivated to do anything & 0.03 & 0.01 & -0.01 & 0.97 & 0.00 & 0.01 & -0.01 & 0.78 \\
\hline 18. Lack of motivation to make appointment & 0.02 & -0.01 & 0.08 & 0.71 & 0.09 & 0.00 & 0.09 & 0.78 \\
\hline \multicolumn{9}{|l|}{ 5. Emotional concerns } \\
\hline 16. Concerns about upsetting feelings in therapy & 0.04 & -0.01 & 0.01 & -0.04 & 0.98 & 0.00 & 0.03 & 0.77 \\
\hline 17. Talking about problems makes them worse & 0.24 & 0.00 & 0.04 & 0.09 & 0.56 & -0.01 & -0.01 & 0.65 \\
\hline 15. Anxiety about going far from home & -0.05 & 0.15 & -0.03 & 0.15 & 0.40 & 0.12 & -0.04 & 0.42 \\
\hline \multicolumn{9}{|l|}{ 6. Access to services } \\
\hline 3. Concerns about cost of therapy & -0.25 & -0.07 & -0.01 & -0.07 & 0.04 & 0.70 & 0.09 & 0.46 \\
\hline 5. Lack of available therapy services in area & 0.06 & 0.06 & 0.04 & 0.02 & 0.03 & 0.66 & -0.04 & 0.56 \\
\hline
\end{tabular}




\begin{tabular}{|c|c|c|c|c|c|c|c|c|}
\hline 6. Not knowing how to find a good therapist & 0.12 & 0.00 & 0.08 & 0.07 & -0.02 & 0.54 & 0.00 & 0.51 \\
\hline 1. Problems with transportation & 0.03 & 0.17 & 0.00 & 0.08 & -0.10 & 0.45 & 0.01 & 0.40 \\
\hline \multicolumn{9}{|l|}{ 7. Time constraints } \\
\hline 4. Interference from daily responsibilities & 0.04 & -0.01 & -0.02 & 0.00 & -0.06 & 0.08 & 0.77 & 0.51 \\
\hline 7. Difficulties getting time off work & -0.04 & 0.03 & 0.07 & 0.05 & 0.06 & -0.02 & 0.63 & 0.51 \\
\hline Eigenvalues & 8.30 & 2.45 & 1.87 & 1.22 & 1.14 & 1.09 & 0.87 & \\
\hline Cumulative variance & 0.16 & 0.24 & 0.33 & 0.40 & 0.48 & 0.55 & 0.60 & \\
\hline Cronbach's alpha (Total $\alpha=.91)$ & .89 & .84 & .82 & .88 & .77 & .69 & .67 & \\
\hline
\end{tabular}

\section{Removed items}

2. Caregiving responsibilities

14. Attending therapy feels too self-indulgent

21. Problems not severe enough for therapy

Note. Items that load onto the corresponding factor are in bold. 
Table 2. Summary of results from Exploratory Factor Analysis of the PBPT scale with a clinical subsample $(n=241)$

\begin{tabular}{|c|c|c|c|c|c|c|c|c|}
\hline & \multicolumn{7}{|c|}{ Factors } & \multirow{2}{*}{$\begin{array}{l}\text { Corrected } \\
\text { item-total } \\
\text { correlation }\end{array}$} \\
\hline & 1 & 2 & 3 & 4 & 5 & 6 & 7 & \\
\hline \multicolumn{9}{|l|}{ 1. Stigma } \\
\hline 23. Talking to someone about personal issues & 0.89 & 0.03 & -0.06 & 0.00 & -0.03 & -0.09 & 0.01 & .73 \\
\hline 24. Concern about being judged by a therapist & 0.69 & -0.07 & 0.21 & 0.03 & 0.07 & 0.06 & -0.01 & .79 \\
\hline 22. Family and/or friends knowing about therapy & 0.55 & -0.03 & -0.07 & -0.11 & 0.03 & 0.10 & 0.11 & .45 \\
\hline 25. Do not think a therapist would truly care about me & 0.54 & -0.02 & 0.30 & 0.07 & 0.01 & 0.01 & -0.04 & .65 \\
\hline 26. Accessing therapy means not able to solve own problems & 0.52 & 0.05 & 0.13 & -0.06 & 0.04 & -0.03 & 0.22 & .59 \\
\hline 20. Discomfort around someone seeing me emotional & 0.49 & 0.00 & 0.05 & 0.10 & 0.30 & 0.00 & -0.03 & .68 \\
\hline \multicolumn{9}{|l|}{ 2. Participation restrictions } \\
\hline 8. Difficulties walking or getting around & 0.01 & 0.91 & -0.06 & -0.01 & -0.01 & -0.08 & 0.07 & .77 \\
\hline 10. Serious illness makes it hard to leave home & 0.08 & 0.82 & 0.03 & -0.05 & 0.01 & 0.00 & -0.03 & .80 \\
\hline 9. Physical symptoms (fatigue, pain, breathing difficulties etc.) & -0.07 & 0.71 & 0.12 & 0.08 & 0.03 & 0.09 & 0.01 & .81 \\
\hline \multicolumn{9}{|l|}{ 3. Negative evaluation } \\
\hline 13. Would not expect therapy to be helpful & 0.00 & 0.00 & 0.85 & -0.01 & -0.04 & -0.04 & 0.11 & .71 \\
\hline 12. Distrust of therapists & 0.21 & 0.09 & 0.60 & -0.05 & 0.07 & 0.13 & -0.03 & .68 \\
\hline 11. Bad past experiences & 0.00 & 0.13 & 0.54 & 0.13 & -0.02 & 0.18 & -0.07 & .59 \\
\hline 14. Attending therapy feels too self-indulgent ${ }^{\dagger}$ & 0.02 & -0.05 & $\mathbf{0 . 5 3}$ & 0.03 & 0.21 & -0.11 & 0.19 & .51 \\
\hline \multicolumn{9}{|l|}{ 4. Lack of motivation } \\
\hline 19. Difficulty getting motivated to do anything & 0.09 & 0.01 & -0.02 & 0.95 & 0.00 & -0.01 & 0.00 & .77 \\
\hline 18. Lack of motivation to make appointment & -0.02 & -0.01 & 0.15 & 0.69 & 0.10 & 0.02 & 0.06 & .77 \\
\hline \multicolumn{9}{|l|}{ 5. Emotional concerns } \\
\hline 16. Concerns about upsetting feelings in therapy & 0.06 & 0.01 & 0.00 & -0.05 & 0.96 & -0.01 & 0.03 & .74 \\
\hline 17. Talking about problems makes them worse & 0.17 & 0.01 & 0.11 & 0.06 & 0.60 & -0.01 & -0.02 & .60 \\
\hline 15. Anxiety about going far from home & -0.08 & 0.17 & -0.05 & 0.15 & 0.42 & 0.11 & -0.04 & .38 \\
\hline \multicolumn{9}{|l|}{ 6. Access to services } \\
\hline 3. Concerns about cost of therapy & -0.15 & -0.09 & -0.07 & -0.08 & 0.05 & 0.72 & 0.04 & .43 \\
\hline 5. Lack of available therapy services in area & 0.13 & 0.04 & 0.03 & 0.04 & 0.09 & 0.56 & 0.04 & .53 \\
\hline
\end{tabular}




\begin{tabular}{|c|c|c|c|c|c|c|c|c|}
\hline 1. Problems with transportation & 0.06 & 0.11 & 0.08 & 0.06 & -0.07 & 0.49 & -0.04 & .41 \\
\hline 6. Not knowing how to find a good therapist & 0.28 & 0.02 & 0.00 & 0.12 & -0.08 & 0.43 & 0.04 & .44 \\
\hline \multicolumn{9}{|l|}{ 7. Time constraints } \\
\hline 7. Difficulties getting time off work & -0.01 & 0.12 & -0.08 & 0.08 & 0.02 & 0.03 & 0.69 & .53 \\
\hline 4. Interference from daily responsibilities & 0.01 & -0.03 & 0.05 & -0.01 & -0.06 & 0.25 & 0.68 & .53 \\
\hline Eigenvalues & 7.46 & 2.55 & 1.94 & 1.26 & 1.39 & 1.18 & 0.91 & \\
\hline Cumulative variance & 0.13 & 0.22 & 0.32 & 0.39 & 0.47 & 0.54 & 0.58 & \\
\hline Cronbach's alpha (Total $\alpha=.89$ ) & .86 & .85 & .80 & .87 & .74 & .67 & .68 & \\
\hline
\end{tabular}

\section{Removed items}

2. Caregiving responsibilities

27. Concerns about medical or insurance records of therapy ${ }^{\dagger}$

21. Problems not severe enough for therapy

Note. Items that load onto the corresponding factor are in bold. Clinical subsample includes participants from the full EFA sample ( $n=354)$ scoring in the clinical or subclinical range on the Questionnaire for Eating Disorder Diagnoses (Q-EDD). "Item interpretation based on factor loadings differed substantively from original EFA shown in Table 1. 
Table 3. Pearson's correlation coefficients for the PBPT, PHQ2 and GHSQ scales $(n=354)$.

\begin{tabular}{|c|c|c|c|c|c|c|c|c|c|c|c|c|}
\hline & \multirow[b]{2}{*}{$\begin{array}{l}\text { PHQ2 } \\
\text { Total }\end{array}$} & \multirow{2}{*}{$\begin{array}{l}\text { GHSQ } \\
\text { Total }\end{array}$} & \multicolumn{3}{|l|}{ GHSQ items } & \multicolumn{7}{|c|}{ PBPT subscales } \\
\hline & & & $\begin{array}{l}\mathrm{MH} \\
\text { professional }\end{array}$ & $\begin{array}{l}\text { Online } \\
\text { treatment }\end{array}$ & $\begin{array}{l}\text { No } \\
\text { treatment }\end{array}$ & Stigma & $\begin{array}{l}\text { Participation } \\
\text { Restrictions }\end{array}$ & $\begin{array}{l}\text { Negative } \\
\text { Evaluation }\end{array}$ & Motivation & $\begin{array}{l}\text { Emotional } \\
\text { Concerns }\end{array}$ & $\begin{array}{l}\text { Access to } \\
\text { Services }\end{array}$ & $\begin{array}{l}\text { Time } \\
\text { Constraints }\end{array}$ \\
\hline PBPT Total & 0.39 & -0.28 & -0.19 & $0.00^{\mathrm{a}}$ & 0.41 & 0.90 & 0.50 & 0.83 & 0.69 & 0.83 & 0.69 & 0.48 \\
\hline PHQ2 Total & 1 & -0.23 & $0.06^{\mathrm{a}}$ & $0.10^{\mathrm{a}}$ & 0.29 & 0.36 & 0.13 & 0.30 & 0.43 & 0.36 & 0.26 & $0.06^{\mathrm{a}}$ \\
\hline GHSQ Total & & 1 & 0.54 & 0.37 & -0.61 & -0.34 & $0.01^{\mathrm{a}}$ & -0.24 & -0.23 & -0.20 & -0.15 & $-0.09^{\mathrm{a}}$ \\
\hline \multicolumn{13}{|l|}{ GHSQ items } \\
\hline MH professional & & & 1 & 0.12 & -0.34 & -0.22 & $-0.01^{\mathrm{a}}$ & -0.19 & -0.13 & -0.12 & -0.11 & $-0.10^{\mathrm{a}}$ \\
\hline Online treatment & & & & 1 & $0.02^{\mathrm{a}}$ & $-0.00^{\mathrm{a}}$ & $0.06^{\mathrm{a}}$ & $-0.01^{\mathrm{a}}$ & $-0.03^{\mathrm{a}}$ & 0.00 & $0.02^{\mathrm{a}}$ & $-0.00^{\mathrm{a}}$ \\
\hline No treatment & & & & & 1 & 0.45 & $0.07^{\mathrm{a}}$ & 0.32 & 0.31 & 0.30 & 0.24 & 0.16 \\
\hline \multicolumn{13}{|l|}{ PBPT subscales } \\
\hline Stigma & & & & & & 1 & 0.26 & 0.69 & 0.57 & 0.74 & 0.50 & 0.36 \\
\hline Participation Restrictions & & & & & & & 1 & 0.39 & 0.33 & 0.38 & 0.34 & 0.18 \\
\hline Negative Evaluation & & & & & & & & 1 & 0.50 & 0.65 & 0.48 & 0.25 \\
\hline Motivation & & & & & & & & & 1 & 0.57 & 0.38 & 0.22 \\
\hline Emotional Concerns & & & & & & & & & & 1 & 0.45 & 0.26 \\
\hline Access to Services & & & & & & & & & & & 1 & 0.45 \\
\hline
\end{tabular}

${ }^{2}$ Correlation not significant at the $p<.05$ level, based on Holm-adjusted significance tests corrected for multiple comparisons (Holm, 1979). 
POPULATIONS

Table 4. PBPT mean subscale scores ranked from highest to lowest

\begin{tabular}{lllll}
\hline PBPT Subscale & Mean & SD & $t$ & $p$ \\
\hline 6. Access to services & 2.65 & 0.82 & 1.616 & .390 \\
7. Time constraints & 2.56 & 1.03 & 4.360 & $<.001$ \\
1. Stigma & 2.31 & 1.02 & 0.138 & .999 \\
4. Lack of motivation & 2.30 & 1.19 & 0.696 & .913 \\
3. Negative evaluation & 2.26 & 1.07 & 2.808 & .027 \\
5. Emotional concerns & 2.10 & 1.02 & 12.874 & $<.001$ \\
2. Participation restrictions & 1.37 & 0.75 & - & - \\
\hline
\end{tabular}

Note. Mean scores computed by dividing sum score by number of items for each subscale. Significance tests reflect $t$-test comparing mean scores between adjacent levels (e.g. 6 vs 7, 7 vs 1); $p$ values adjusted for multiple tests using the Dunnett correction for correlated estimates. 


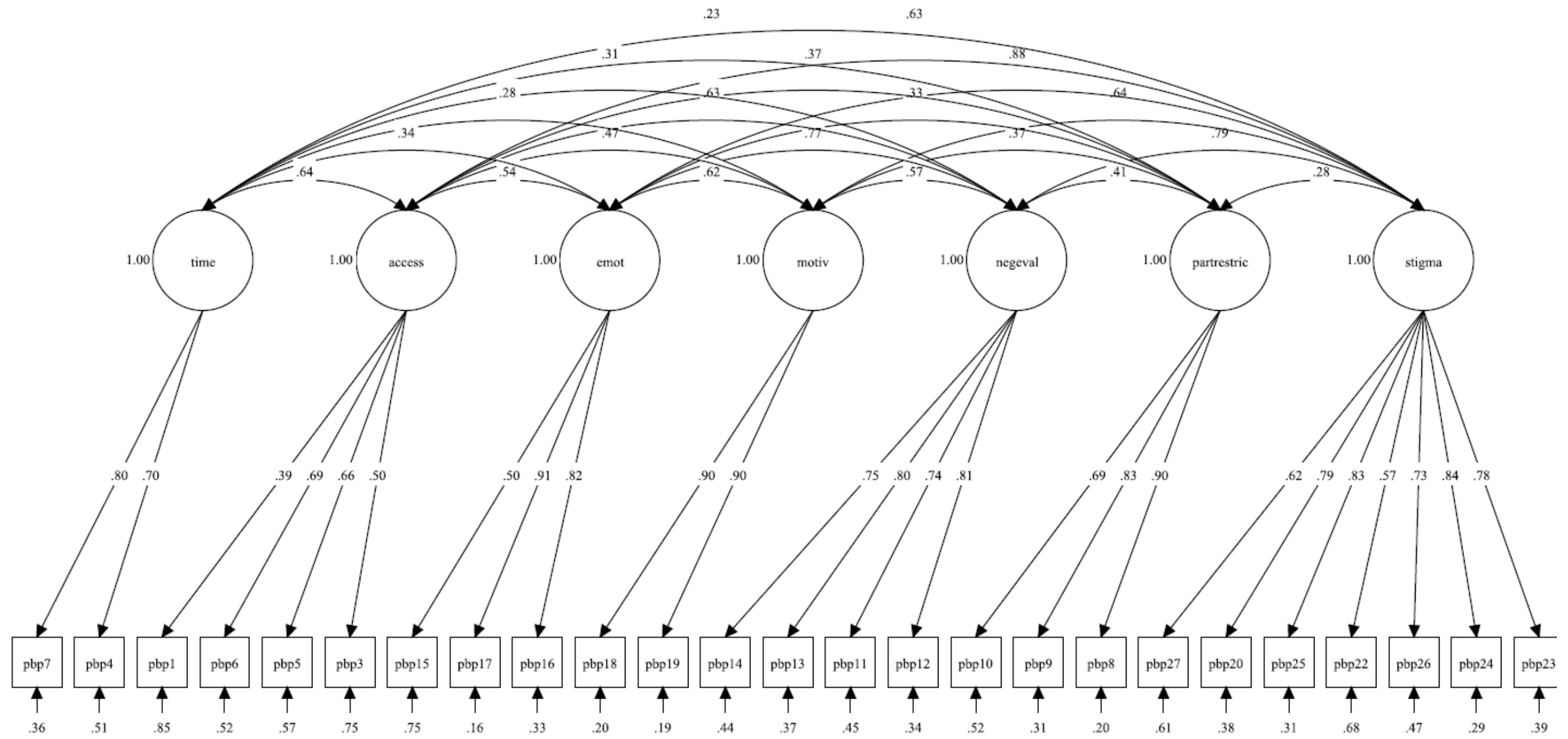

Figure 1. CFA measurement model for the PBPT showing standardized coefficients; $\chi^{2}(254)=587.65, p<.001, \mathrm{CFI}=0.912, \mathrm{RMSEA}=0.061[95 \%$ CI: $0.054,0.067], \mathrm{SRMR}=0.058$ 
POPULATIONS 